\title{
Serum proteomic analysis identifies sex-specific differences in lipid metabolism and inflammation profiles in adults diagnosed with Asperger syndrome
}

Hannah Steeb ${ }^{1}$, Jordan M Ramsey ${ }^{1}$, Paul C Guest ${ }^{1}$, Pawel Stocki ${ }^{1}$, Jason D Cooper ${ }^{1}$, Hassan Rahmoune ${ }^{1}$, Erin Ingudomnukul ${ }^{2}$, Bonnie Auyeung ${ }^{2}$, Liliana Ruta ${ }^{3}$, Simon Baron-Cohen ${ }^{2}$ and Sabine Bahn ${ }^{1,4^{*}}$

\begin{abstract}
Background: The higher prevalence of Asperger Syndrome (AS) and other autism spectrum conditions in males has been known for many years. However, recent multiplex immunoassay profiling studies have shown that males and females with AS have distinct proteomic changes in serum.

Methods: Here, we analysed sera from adults diagnosed with AS (males $=14$, females $=16$ ) and controls (males $=13$, females $=16$ ) not on medication at the time of sample collection, using a combination of multiplex immunoassay and shotgun label-free liquid chromatography mass spectrometry (LC-MS ${ }^{\mathrm{E}}$ ). The main objective was to identify sex-specific serum protein changes associated with AS.

Results: Multiplex immunoassay profiling led to identification of 16 proteins that were significantly altered in AS individuals in a sex-specific manner. Three of these proteins were altered in females (ADIPO, IgA, APOA1), seven were changed in males (BMP6, CTGF, ICAM1, IL-12p70, IL-16, TF, TNF-alpha) and six were changed in both sexes but in opposite directions (CHGA, EPO, IL-3, TENA, PAP, SHBG). Shotgun LC-MSE profiling led to identification of 13 serum proteins which had significant sex-specific changes in the AS group and, of these, 12 were altered in females (APOC2, APOE, ARMC3, CLC4K, FETUB, GLCE, MRRP1, PTPA, RN149, TLE1, TRIPB, ZC3HE) and one protein was altered in males (RGPD4). The free androgen index in females with AS showed an increased ratio of 1.63 compared to controls.

Conclusion: Taken together, the serum multiplex immunoassay and shotgun LC-MSE profiling results indicate that adult females with AS had alterations in proteins involved mostly in lipid transport and metabolism pathways, while adult males with AS showed changes predominantly in inflammation signalling. These results provide further evidence that the search for biomarkers or novel drug targets in AS may require stratification into male and female subgroups, and could lead to the development of novel targeted treatment approaches.
\end{abstract}

Keywords: Asperger Syndrome, Sex, Proteomics, Biomarkers, Lipid transport, Growth, Inflammation

\footnotetext{
* Correspondence: sb209@cam.ac.uk

'Department of Chemical Engineering \& Biotechnology, University of

Cambridge, Tennis Court Road, Cambridge, UK

${ }^{4}$ Department of Neuroscience, Erasmus Medical Centre, Rotterdam, Netherlands

Full list of author information is available at the end of the article
} waiver (http://creativecommons.org/publicdomain/zero/1.0/) applies to the data made available in this article, unless otherwise stated. 


\section{Background}

Asperger syndrome (AS) is a subgroup within autism spectrum conditions (ASCs) [1]. The prevalence of AS in the general population is about $1 \%[2,3]$. ASC leads to impairments in reciprocal social interaction and communication, alongside unusually restricted interests and repetitive behaviour [4], although people with AS develop language at the normal age and have no general cognitive delay. Similar to other forms of ASC, the prevalence of AS is higher in males compared to females with an approximate 4:1 ratio [5]. This suggests that sex-specific differences may affect its susceptibility, aetiology and/or manifestation.

In line with this, previous multiplex immunoassay profiling studies targeting specific classes of proteins have identified diagnosis-sex interactions in serum molecules such as cytokines, steroid and metabolic hormones, growth factors and lipid transport in adults with AS [6,7]. In these studies, females with AS had a higher number of changes in the levels of lipid and hormone-related molecules, and males with AS showed more changes in molecules related to dysfunction of immune or inflammatory pathways. However, individuals with AS often present with co-morbid psychiatric, neurological, gastrointestinal, metabolic, cardiovascular, gynaecological or musculoskeletal conditions [8]. This can make interpretation of the results of proteomic analyses difficult as the findings may be affected by drug- and lifestyle-related confounding factors.

Here, we attempt to minimize this possibility by analyzing samples from people with AS $(n=30)$ and controls $(n=29)$ who were not on medication at the time of blood collection and accounting for daily lifestyle routines. In addition, we used a combination of multiplex immunoassay and shotgun liquid chromatography mass $\left(\mathrm{LC}-\mathrm{MS}^{\mathrm{E}}\right)$ profiling platforms to increase the analytical coverage to a wider range of protein classes [9]. The main objective was to identify sex-specific protein alterations in serum from people with AS compared to controls.

\section{Methods}

\section{Clinical samples}

Informed written consent was given by all participants. The protocols were approved by the UK National Health Service Cambridge Research Ethics Committee and studies were carried out in accordance with the Declaration of Helsinki. Recruitment of participants with AS was carried out as described by Schwarz et al. [6] and diagnoses were made by clinical psychologists or psychiatrists based on Diagnostic and Statistical Manual of Mental Disorders IV-Text Review (DSM-IV-TR). All participants completed the Autism Spectrum Quotient (AQ) and the Empathy Quotient (EQ) forms [10]. Participants with a family history of serious mental illness or metabolic, cardiovascular or inflammatory diseases were excluded to minimise these as potential confounding factors. Samples from people with AS or controls who were not taking medications (antidepressants, antipsychotics, immunosuppressants, insulin) or using tobacco or marijuana at the time of sample collection were used in the current study to minimise the possibility of detecting drug-related proteomic changes (Table 1). Lifestyle information such as exercise level, alcohol intake, and oral contraception in females was also documented (Table 1).

\section{Sample collection}

Blood samples were collected into $7.5 \mathrm{~mL}$ S-Monovette serum tubes (Sarstedt; Numbrecht, Germany) and placed at room temperature for two hours to allow coagulation, according to standard protocols. After this, the tubes were centrifuged at $1,100 \times \mathrm{g}$ for ten minutes to pellet the clotted material and other debris. The resulting serum supernatants were transferred into LoBind Eppendorf tubes (Hamburg, Germany) and stored at $-80^{\circ} \mathrm{C}$.

\section{Multiplex immunoassay analysis}

Serum samples from drug-free people with AS $(n=30)$ and controls $(n=29)$ were analyzed using the HumanMAP panel comprised of immunoassays for 119 analytes (Additional file 1: Table S1) in a Clinical Laboratory Improved Amendments-certified laboratory at Myriad-RBM (Austin, TX, USA) as described previously [6,11]. The assays were calibrated using duplicate standard curves of each analyte and raw intensity measurements converted to protein concentrations using proprietary software. All measurements were conducted using randomized samples under blind conditions to minimize biases or batch effects.

\section{Mass spectrometry analysis}

Depletion of abundant proteins was carried out in $40 \mu \mathrm{L}$ of serum using the Human 14 Affinity Removal System (Agilent Technologies, Santa Rosa, CA, USA) on the AKTA purifier system (GE Healthcare, Uppsala, Sweden). This was carried out to increase the detection of higher numbers of low abundance proteins that are potentially masked by the more abundant serum components. The flow through fractions containing the low abundance proteins were exchanged into $50 \mathrm{mM}$ ammonium bicarbonate (pH 8.0) using pre-washed $5 \mathrm{kDa}$-molecular weight cut-off Centricon tubes (Agilent Technologies; Santa Rosa, CA, USA). Protein concentrations were determined using the Biorad DC protein assay according to standard protocols (Hercules, CA, USA). In order to reduce disulfide bonds on proteins, samples were incubated for 30 minutes at $60^{\circ} \mathrm{C}$ with $100 \mathrm{mM}$ dithiothreitol (Sigma Aldrich; Poole, UK). After this, $200 \mathrm{mM}$ iodoacetamide $(2.63 \mu \mathrm{L}$; Sigma Aldrich, Poole, UK) was added to each sample to alkylate the reduced cysteine residues, by incubation in the dark 
Table 1 Demographics of non-medicated people with Asperger syndrome (AS) and controls used in the study

\begin{tabular}{|c|c|c|c|c|}
\hline & \multicolumn{2}{|c|}{ Male } & \multicolumn{2}{|c|}{ Female } \\
\hline & Patient & Control & Patient & $\overline{\text { Control }}$ \\
\hline Sample number & 14 & 13 & 16 & 16 \\
\hline Age (years) & $31 \pm 9$ & $31 \pm 6$ & $33 \pm 9$ & $34 \pm 5$ \\
\hline BMI $\left(\mathrm{kg} / \mathrm{m}^{2}\right)$ & $24 \pm 3$ & $25 \pm 4$ & $26 \pm 5$ & $25 \pm 6$ \\
\hline Smoking (yes/no) & $2 / 12$ & $3 / 10$ & $4 / 12$ & $2 / 14$ \\
\hline Exercise level & $5 / 4 / 5 / 0 / 0$ & $8 / 3 / 2 / 0 / 0$ & $1 / 2 / 10 / 2 / 1$ & $6 / 2 / 7 / 1 / 0$ \\
\hline Alcohol & $2 / 3 / 0 / 0 / 4 / 5$ & $6 / 1 / 0 / 2 / 1 / 3$ & $1 / 1 / 0 / 0 / 3 / 11$ & $5 / 5 / 2 / 2 / 1 / 1$ \\
\hline Oral contraception & NA & NA & $7 / 9$ & $4 / 12$ \\
\hline AQ score & $38 \pm 13$ & $15 \pm 6$ & $39 \pm 13$ & $14 \pm 5$ \\
\hline EQ score & $28 \pm 14$ & $37 \pm 14$ & $15 \pm 16$ & $52 \pm 10$ \\
\hline
\end{tabular}

Values are represented as mean \pm standard deviation. BMI = body mass index. Exercise: high activity/moderate activity/low activity/sedentary/NA. Alcohol: 1 to 5 units/6 to 10 units/11 to 15 units/16 to 20 units/none/NA. Oral contraception: never/past (not current).

for 30 minutes at room temperature. Proteins were then digested using sequencing grade modified trypsin (Promega; Madison, WI, USA) at a ratio of 1:50 (w/w trypsin/protein) for 17 hours at $37^{\circ} \mathrm{C}$. Digestions were stopped by addition of 1:60 $8.8 \mathrm{M} \mathrm{HCl}$ to each sample. Samples were stored at $-80^{\circ} \mathrm{C}$ prior to $\mathrm{LC}-\mathrm{MS}^{\mathrm{E}}$ analysis.

All solvents used for chromatography were of mass spectrometry grade (Fisher Scientific; Loughborough, UK). Buffers used were (A) $0.1 \%$ formic acid in water and (B) $0.1 \%$ formic acid in acetonitrile. Samples were diluted with buffer A to a final $0.12 \mu \mathrm{g} / \mu \mathrm{L}$ protein concentration and injected into the system. Each sample was analysed twice followed by alternating injections of a blank or a standard of $25 \mathrm{fmol} / \mu$ Ltryptically-digested yeast enolase (Waters Corporation; Milford, MA, USA). The samples were analysed on a nanoAquity ultra-performance liquid chromatography quadrupole time-of-flight (UPLCQTOF) Premier mass spectrometer (Waters Corporation, Elstree, UK) with a gradient starting at $97 \%$ buffer A (3\% buffer B), followed by ramping to $70 \% \mathrm{~A}$ in 80 minutes, $70 \%$ to $5 \%$ in $10 \mathrm{mi}$ nutes, running isocratically at $5 \% \mathrm{~A}$ for 10 minutes, then returning to initial conditions over 1 minute. The analytical column was coupled through a $10 \mu \mathrm{m}$ fused-silica emitter (New Objective; Woburn, MA, USA) to the mass spectrometer, which was operated in positive $\mathrm{V}$ mode (resolution: 10,000 full width at half-maximum). The alternative scanning, data-independent expression mode $\left(\mathrm{LC}-\mathrm{MS}^{\mathrm{E}}\right.$ ) was achieved with a setting for the low collision energy of $5 \mathrm{eV}$ and the high collision energy ramped between 15 and $42 \mathrm{eV}$ per scan. Acquisition time in each function was 0.6 seconds. Argon was used as the collision gas. Molecular ions were mass-corrected using the monoisotopic mass of the doubly-charged precursor of glufibrinopeptide B (785.8426 mass/charge), which was infused continuously using a reference spray apparatus.

The ProteinLynx Global Server (PLGS, version 2.4; Waters Corporation, Elstree, UK) was used for smoothing, centring, de-isotoping and charge state reduction of mass spectral peaks. Peptide fragment ions were allocated to peptide precursor ions based on identical retention times and elution profiles. For protein identification, an algorithm described by Li and colleagues was for searching the human Swiss-Prot database version 57.4 [12]. Time alignment was accomplished using the Elucidator ${ }^{\mathrm{TM}}$ software (Rosetta Biosoftware; Seattle, WA, USA) by applying the PeakTeller algorithm [13]. Peptide and fragment ion intensities were normalised to the total ion current and this required detection of these ions in both technical replicates of each sample and in at least $67 \%$ of the samples within each group. Protein intensities were calculated by summing the intensities of all peptide ions (mean values of technical replicates) associated with specific proteins.

\section{Statistical analysis}

Principal component analysis (PCA) was carried out using the software SIMCA P+, v 2.12 (Umetrics; Stockholm, Sweden) to identify potential outliers in mass spectrometry data. PCA showed no clustering of the data based on demographic variables (data not shown). However, samples did show clustering based on analysis order. Therefore, protein intensities in mass spectrometry data were normalised using the median intensity of each batch to remove this effect. All other statistical tests were conducted using the free statistical software package R, v. 2.15.0 (www.r-project.org). Multiplex immunoassay data were first pre-processed by removing analytes containing more than $30 \%$ missing values, resulting in 33 analytes being discarded and leaving 114 for analysis. The proportion of missing values in this dataset was less than $2 \%$. Remaining missing values were imputed with twice the maximum or half the minimum analyte concentrations for measurements above and below the limits of quantitation, respectively. Missing values resulting from 
insufficient sample volume were replaced by the analyte mean. Both multiplex immunoassay and MS data were $\log _{\mathrm{e}}$ transformed to stabilise variance and improve normality and outliers outside three standard deviations of the mean were removed. Differences in molecular levels between individuals with AS and control individuals were assessed for each analyte using stepwise regression, with sex, age, BMI, and exercise as additional covariates. $P$-values were adjusted to control the false discovery rate (FDR). Sex-diagnosis interactions were analysed in the same manner for each analyte. Those analytes with significant sex-diagnosis interactions $(P<0.05)$ were reanalysed for males and females separately and classified as being changed in females with AS (female-specific), changed in males with AS (male-specific), or in both with opposing directional changes (qualitative interaction). Analytes with changes of less than 10\% were discarded. We also carried out Spearman correlation analyses in order to determine whether any of the measured serum analytes were correlated with AQ or EQ scores.

\section{Single reaction monitoring (SRM) mass spectrometry} Candidate proteins identified by label free $\mathrm{LC}-\mathrm{MS}^{\mathrm{E}}$ profiling were retested using single-reaction monitoring (SRM) on a XevoTQ-S mass spectrometer (Waters Corporation, Elstree, UK) coupled to a nanoAcquityUPLC system (Waters Corporation, Elstree, UK) as described previously [14]. This was aimed at providing a technical replication of the findings. Criteria for selecting candidate peptides representing the corresponding proteins for validation were based on peptide count, uniqueness and quality of transitions. Three peptides were selected for each target protein and isotopically-labelled peptides synthesised at JPT Peptide Technologies $\mathrm{GmbH}$ (Berlin, Germany). Data analyses were performed using the R-package SRM stats [15]. The settings used for group comparison were 'restricted biological replication' and 'expanded technical replication'.

\section{In silico pathway analysis}

The UniProt accession codes of proteins that showed diagnosis-sex interactions were uploaded into the Ingenuity Pathways Knowledge Database (IPKB; Ingenuity ${ }^{\text {बтм }}$ Systems; Mountain View, CA, USA). The pathways most significant to the dataset were determined by automated overlay of the identified proteins onto predefined pathway maps in the IPKB. Fisher's right-tailed exact test was used to calculate $P$ values associated with the identified pathways. The significance of the association between the dataset and canonical pathways was measured by the ratio of the number of significant molecules divided by the total number of molecules in the canonical pathway and by the Fisher's exact test $P$ value.

\section{Results}

\section{Multiplex immunoassay}

Multiplex immunoassay profiling of serum samples resulted in identification of 16 analytes that were present at significantly different levels between drug-free individuals with AS $(n=30)$ and controls $(n=29)$ after adjustment for age, BMI, and exercise (Table 2). The analytes showing the largest ratiometric differences included neuronal cell adhesion molecule that was increased with a ratio of 1.4 in AS compared to controls, and fatty acid binding protein and growth hormone that were decreased with ratios less than 0.5 .

We then identified 16 serum proteins changed in a sex-specific manner in AS. Seven proteins (BMP6, TNF, TF, CTGF, IL-16, IL-12p70, ICAM-1) were increased specifically between males with AS $(\mathrm{n}=14)$ and male controls $(\mathrm{n}=13)$ and three proteins (ADIPO, IgA, APOA1) were decreased in females with AS $(n=16)$ in comparison to female controls $(n=16)$ (Figure 1 and Table 3$)$. In addition, six proteins (CHGA, TENA, SHBG, PAP, EPO, IL-3) showed opposite-increased or -decreased concentrations between the AS male and AS female groups. In the latter case, the differences for SHBG $(P=0.065)$ and EPO $(P=0.060)$ did not reach significance between females with AS and female controls (Figure 1 and Table 3). BMP6 showed the highest male-specific increase in AS compared to controls at a ratio of 3.04 and IL-3 showed the strongest decrease with a ratio of 0.28 . Conversely, IL-3 showed

Table 2 Identification of analytes altered between individuals with Asperger syndrome (AS) $(n=30)$ and controls $(n=29)$ using multiplex immunoassay analysis

\begin{tabular}{lccc}
\hline & $P$-value & FDR & Ratio \\
\hline Neuronal cell adhesion molecule & 0.022 & 0.19 & 1.40 \\
IL-5 & 0.007 & 0.13 & 1.28 \\
CD40 & 0.008 & 0.13 & 1.21 \\
Cortisol & 0.003 & 0.088 & 1.20 \\
TNF-alpha & 0.024 & 0.19 & 1.20 \\
IL-7 & 0.012 & 0.15 & 1.18 \\
BDNF & 0.015 & 0.17 & 1.16 \\
Sortilin & 0.007 & 0.13 & 1.15 \\
Serum glutamic-oxaloacetic transaminase & 0.003 & 0.088 & 0.82 \\
Apolipoprotein A1 & 0.018 & 0.19 & 0.79 \\
Immunoglobulin M & 0.002 & 0.088 & 0.73 \\
HB-epidermal growth factor & 0.002 & 0.088 & 0.70 \\
Eotaxin-3 & 0.048 & 0.29 & 0.60 \\
Ferritin & 0.010 & 0.14 & 0.60 \\
Fatty acid binding protein & 0.030 & 0.20 & 0.50 \\
Growth hormone & 0.029 & 0.20 & 0.39 \\
\hline The iesuls are adusted for age and BMI.FDR & &
\end{tabular}

The results are adjusted for age and BMI. FDR = false discovery rate. Ratio $=$ AS/control. Grey shading indicates these proteins had a significant sex-diagnosis interaction. 


\begin{tabular}{|c|c|c|}
\hline \multirow{3}{*}{$\begin{array}{c}\text { Lipid } \\
\text { metabolism }\end{array}$} & FEMALES & MALES \\
\hline & 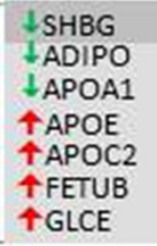 & $\uparrow S H B G$ \\
\hline & $\begin{array}{l}\text { EPO } \\
\text { ¿CHGA } \\
\text { \&PAP }\end{array}$ & 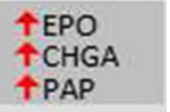 \\
\hline \multirow[t]{3}{*}{$\begin{array}{l}\text { Cell growth } \\
\text { proliferation }\end{array}$} & 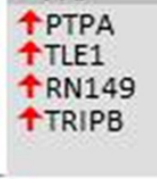 & ^BMP6 \\
\hline & $\begin{array}{l}\text { \IL-3 } \\
\text { ITENA }\end{array}$ & $\begin{array}{l}\text { 个IL-3 } \\
\text { 个TENA }\end{array}$ \\
\hline & TCLC $4 \mathrm{~K}$ & 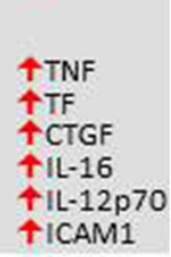 \\
\hline $\begin{array}{l}\text { Nucleic acid } \\
\text { processing }\end{array}$ & $\begin{array}{l}\downarrow \mathrm{MRRP} 1 \\
\uparrow \mathrm{ZC} 3 \mathrm{HE}\end{array}$ & \\
\hline Other & TARMC 3 & $\downarrow R G P D 4$ \\
\hline \multicolumn{3}{|c|}{$\begin{array}{l}\text { Figure } 1 \text { Sex-specific and common changes in people with } \\
\text { Asperger syndrome (AS) compared to controls. The red arrows } \\
\text { indicate an increase and the green arrows show a decrease of the } \\
\text { protein in AS relative to controls. The proteins in grey boxes are } \\
\text { changed in both males in females but in opposite directions. The } \\
\text { abbreviations are as indicated in Table } 2 \text {. }\end{array}$} \\
\hline
\end{tabular}

the highest female-specific increase with a ratio of 1.87 and APOA1 showed the greatest decrease at a ratio of 0.63 (Table 3).

We found that the levels of SHBG were decreased in females with AS compared to both males with AS and controls, which could be associated with higher levels of free testosterone. We estimated the free testosterone levels in both males and females by dividing total testosterone (measured by the multiplex immunoassay panel) by the SHBG levels. This is termed the free androgen index (FAI). In females, the FAI showed an increased ratio of $1.63(P=0.0275)$ in individuals with AS compared to controls. In males, the FAI was found at a ratio of 0.85 in AS compared to controls although this was not significant $(P=0.2206)$. Given the prior association of increased testosterone-related medical conditions in adult females with ASC [16], we tested the levels of testosterone, SHBG and the FAI in relation to AQ and the EQ scores in AS using Spearman correlation analysis. However, this revealed no significant correlations.

\section{Mass spectrometry}

LC-MS ${ }^{\mathrm{E}}$ proteome profiling of serum was performed to identify novel gender-specific serum biomarkers not analysed using the multiplex immunoassay platform. Using LC-MS ${ }^{\mathrm{E}}$ we measured the levels of 9,068 serum peptides, which corresponded to 313 proteins using the criteria outlined in the methods section. We found 13 proteins with significant sex-diagnosis interactions, 12 of which (ARMC3, PTPA, TLE1, CLC4K, GLCE, APOC2, ZC3HE, FETUB, RN149, TRIPB, APOE, MRRP1) were altered specifically in females with AS compared to female controls. Only one protein (RGPD4) was altered only in males with AS compared to male controls (Figure 1, Table 4).

ARMC3 and PTPA showed the highest increases in females with AS compared to female controls at ratios of 1.29 and 1.23, respectively. MRRP1 showed the greatest decrease in females with AS compared to female controls at a ratio of 0.78. The finding of increased APOE levels in females with AS compared to female controls was the most significant result in this study $(P=0.0002)$. Because the FDR for APOE was 0.72, we attempted to validate the findings using an orthogonal analysis as described in the methods section. An SRM mass spectrometry assay was established for APOE and this confirmed that it was increased in females with AS compared to female controls with a ratio change of $1.27\left(P=2.13 \mathrm{E}^{-13}\right)$, which was more robust than the findings for the LC-MS ${ }^{\mathrm{E}}$ study (1.11 fold) (Figure 2).

\section{In silico pathway analysis}

The Uniprot accession codes for 19 proteins associated with females with AS were uploaded into the IPKB to identify the most significant networks, diseases and canonical pathways associated with the dataset. Note that no code for IgA was uploaded as this was not present in the database. A single network was identified which showed interactions for nine of these proteins (ADIPO, APOA1, APOC2, APOE, EPO, IL-3, PAP, SHBG, TENA) and the predominant function associated with these proteins was lipid metabolism. The most significant disease was listed as 'cancer', although this was due to the effects on cell proliferation $(P=5.9 \mathrm{E}-06$ - 5.0E-02), accounted for by nine proteins (ADIPO, APOA1, APOE, ARMC3, CHGA, EPO, IL-3, PAP, TENA). The most significant canonical pathway was FXR/RXRsignalling (1.9E-06) which was covered by four proteins (APOA1, APOC2, APOE, FETUB). Of the 13 male AS-associated proteins, nine of these (BMP6, CTGF, EPO, ICAM1, IL-3, IL-16, SHBG, TENA, TNF) were associated with a single network with a predominant function of cell to cell signalling. The top disease was inflammation signalling $(P=2.0 \mathrm{E}-06-4.2 \mathrm{E}-03$; seven proteins: EPO, ICAM1, IL-3, IL-12p70, IL-16, TENA, TNF) and the top canonical pathway was differential regulation of cytokine production $(P=3.8 \mathrm{E}-07$; three proteins: IL-3, IL-12p70, TNF). 
Table 3 Summary of significant sex $x$ diagnosis interactions of serum molecules measured by multiplex immunoassay

\begin{tabular}{|c|c|c|c|c|c|c|}
\hline \multirow[b]{2}{*}{ Protein } & \multicolumn{2}{|c|}{ Interaction } & \multicolumn{2}{|c|}{ Males } & \multicolumn{2}{|c|}{ Females } \\
\hline & $P$-value & q-value & Ratio & $P$-value & Ratio & $P$-value \\
\hline Bone morphogenic protein-6 (BMP6) & 0.023 & 0.193 & 3.04 & 0.001 & 0.85 & 0.703 \\
\hline Tumour necrosis factor-alpha (TNF) & 0.017 & 0.191 & 1.45 & 0.003 & 1.02 & 0.192 \\
\hline Tissue factor (TF) & 0.012 & 0.154 & 1.39 & 0.001 & 0.73 & 0.151 \\
\hline Connective tissue growth factor (CTGF) & 0.001 & 0.037 & 1.29 & 0.001 & 0.83 & 0.091 \\
\hline Interleukin-16 (IL-16) & 0.025 & 0.196 & 1.17 & 0.008 & 0.88 & 0.134 \\
\hline Interleukin-12p70 (IL-12p70) & 0.005 & 0.070 & 1.16 & 0.008 & 0.91 & 0.074 \\
\hline Intracellular adhesion molecule-1 (ICAM1) & 0.027 & 0.196 & 1.15 & 0.010 & 1.00 & 0.343 \\
\hline Chromogranin A (CHGA) & $<0.001$ & 0.008 & 1.70 & 0.001 & 0.77 & 0.019 \\
\hline Tenascin C (TENA) & 0.002 & 0.037 & 1.28 & 0.049 & 0.66 & 0.007 \\
\hline Sex hormone binding globulin (SHBG) & 0.026 & 0.191 & 1.20 & 0.036 & 0.76 & 0.065 \\
\hline Prostatic acid phosphatase (PAP) & 0.004 & 0.058 & 1.19 & 0.040 & 0.82 & 0.028 \\
\hline Erythropoietin (EPO) & 0.044 & 0.282 & 0.49 & 0.021 & 1.36 & 0.060 \\
\hline Interleukin-3 (IL-3) & $<0.001$ & 0.022 & 0.28 & 0.006 & 1.87 & 0.039 \\
\hline Adiponectin (ADIPO) & 0.037 & 0.251 & 1.19 & 0.453 & 0.77 & 0.037 \\
\hline Immunoglobulin A (IgA) & 0.022 & 0.192 & 1.05 & 0.852 & 0.73 & 0.006 \\
\hline Apolipoprotein A1 (APOA1) & 0.002 & 0.037 & 1.05 & 0.857 & 0.63 & 0.001 \\
\hline
\end{tabular}

Indicated are the $P$-values and FDR (false discovery rate) of the sex-diagnosis interactions and the separate ratio (AS/control; calculated using geometric means) changes with $P$-values in males in females. Values in bold font indicate significant sex differences in individuals with AS and typical individuals. Analytes in italic style font were identified in our previous study of AS individuals, which did not account for potential drug effects [6]. In the overlapping region, changes in molecular levels are in opposite directions in males and females.

\section{Discussion}

This is the first molecular profiling study using a combination of multiplex immunoassay and mass spectrometry to investigate sex-specific differences in serum from adults with AS compared to typical controls. All participants were drug-free at the time of sample collection. Specifically, samples were not analysed from individuals taking medications or substances such as antidepressants, antipsychotics, immunosuppressants, antidiabetics, tobacco or marijuana. This study design increases the chances that the identified findings are linked to the underlying pathways altered in AS and minimises possible confounding effects of drug treatment. Analysis of the combined cohort led to identification of 16 analytes that were present at

Table 4 Summary of significant sex-diagnosis interactions of serum molecules measured by LC-MS ${ }^{\mathrm{E}}$ profiling

\begin{tabular}{llcccccc}
\hline & & & & \multicolumn{2}{c}{ Males } & \multicolumn{2}{c}{ Females } \\
\cline { 5 - 6 } Code & Protein & Interaction & FDR & Ratio & $\boldsymbol{P}$-value & Ratio & $\boldsymbol{P}$-value \\
\hline RGPD4 & RANBP2-like and GRIP domain containing 5 & 0.015 & 0.723 & $\mathbf{0 . 8 9}$ & $\mathbf{0 . 0 1 6}$ & 1.07 & 0.893 \\
ARMC3 & Armadillo repeat containing 3 & 0.002 & 0.291 & 0.88 & 0.183 & $\mathbf{1 . 2 9}$ & $\mathbf{0 . 0 0 1}$ \\
PTPA & PP 2A activator, reg subunit 4 & 0.038 & 0.723 & 0.97 & 0.681 & $\mathbf{1 . 2 3}$ & $\mathbf{0 . 0 0 1}$ \\
TLE1 & Transducin-like enhancer of split 1 & 0.049 & 0.723 & 0.90 & 0.394 & $\mathbf{1 . 2 2}$ & $\mathbf{0 . 0 2 6}$ \\
CLC4K & CD207 molecule, langerin & 0.017 & 0.723 & 0.96 & 0.720 & $\mathbf{1 . 2 0}$ & $<\mathbf{0 . 0 0 1}$ \\
GLCE & Glucuronic acid epimerase & 0.017 & 0.723 & 0.88 & 0.231 & $\mathbf{1 . 1 9}$ & $\mathbf{0 . 0 1 8}$ \\
APOC2 & Apolipoprotein C2 & 0.005 & 0.522 & 0.92 & 0.254 & $\mathbf{1 . 1 9}$ & $\mathbf{0 . 0 0 1}$ \\
ZC3HE & Zinc finger CCCH-type containing 14 & 0.039 & 0.723 & 0.97 & 0.383 & $\mathbf{1 . 1 6}$ & $\mathbf{0 . 0 2 0}$ \\
FETUB & Fetuin B & 0.035 & 0.723 & 0.96 & 0.572 & $\mathbf{1 . 1 5}$ & $\mathbf{0 . 0 0 4}$ \\
RN149 & Ring finger protein 149 & 0.044 & 0.723 & 1.02 & 0.588 & $\mathbf{1 . 1 4}$ & $\mathbf{0 . 0 4 2}$ \\
TRIPB & Thyroid hormone receptor interactor 11 & 0.012 & 0.723 & 0.94 & 0.610 & $\mathbf{1 . 1 3}$ & $\mathbf{0 . 0 2 7}$ \\
APOE & Apolipoprotein E & 0.030 & 0.723 & 0.99 & 0.851 & $\mathbf{1 . 1 1}$ & $<\mathbf{0 . 0 0 1}$ \\
MRRP1 & RNA G9 methyltransferase domain cont 1 & 0.044 & 0.723 & 1.06 & 0.592 & $\mathbf{0 . 7 8}$ & $\mathbf{0 . 0 3 5}$ \\
\hline
\end{tabular}

Indicated are the $P$-values and FDR (false discovery rate) of the interactions and the separate ratio (AS/control; calculated using geometric means) changes with $P$-values in males in females. Values in bold font indicate significant differences between AS and typical individuals by sex. 


\section{APOE levels in females}

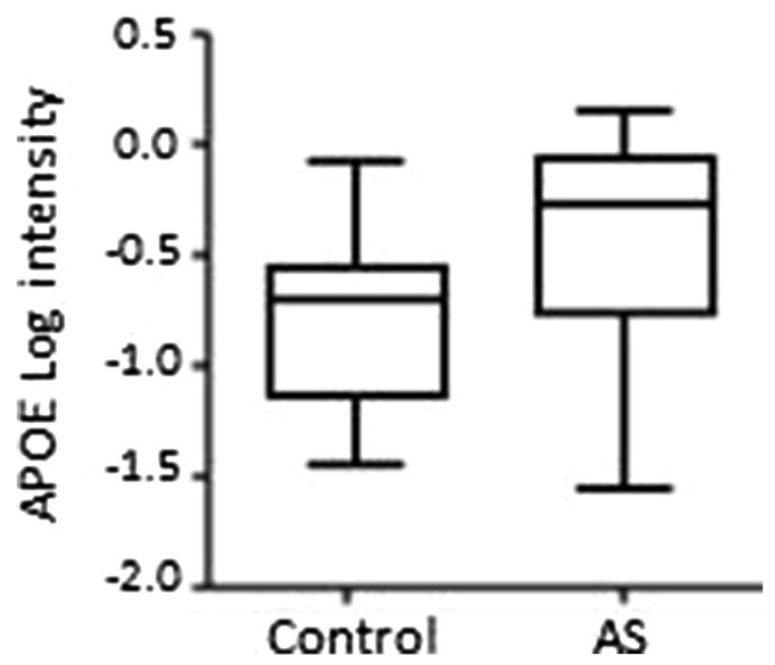

Figure 2 Validation of changes in apolipoprotein E levels in females with Asperger syndrome (AS) $(n=16)$ compared to female controls $(n=16)$ using Selective Reaction Monitoring (SRM) mass spectrometry.

significantly different levels in AS compared to controls. Several of these molecules have been implicated previously in ASC, such as neuronal cell adhesion molecule [17], cortisol [18], TNF-alpha [19], brain-derived neurotrophic factor [20], IL-5 [21], eotaxin-3 [22], immunoglobulin M [23], ferritin [24] and growth hormone [25]. In the next phase of the study, serum samples from approximately equal numbers of males and females were analysed to allow determination of sex-specific changes. This resulted in identification of 29 proteins with significant sex-diagnosis interactions. Fifteen of these (ADIPO, APOA1, APOC2, APOE, ARMC3, CLC4K, FETUB, GLCE, IgA, MRRP1, PTPA, RN149, TLE1, TRIPB, ZC3HE) showed altered levels specifically in female patients, eight (BMP6, CTGF, IL-16, IL-12p70, ICAM-1, RGPD4, TF, TNF) were altered in males, and six (CHGA, TENA, SHBG, PAP, EPO, IL-3) showed opposite changes in females and males. For the multiplex immunoassay analysis, the changes in 11 proteins (APOA1, CHGA, CTGF, EPO, IL-3, IL-12p70, ICAM1, SHBG, TENA, TF and TNF) were consistent with those found in our previous study [6]. This suggests that the sex-specific alterations in these molecules were not influenced by drug effects.

In silico pathway analysis revealed that the predominant pathway affected in females with AS was lipid metabolism. This is in line with previous studies showing alterations in circulating lipids such as cholesterol in individuals with ASC $[26,27]$. All steroids are synthesized from cholesterol and, in the brain, these are involved in regulation of neuronal processes such as $\mathrm{GABA}_{\mathrm{A}}$ and
NMDA receptor signalling, myelin formation and synaptogenesis [28]. This may be of relevance to the current findings since diseases marked by impaired cholesterol biosynthesis, such as Smith-Lemli-Opitz Syndrome (SLOS), are associated with an increased incidence of ASC [29]. Furthermore, treatment of SLOS patients with cholesterol leads to fewer autistic behaviours, infections, and symptoms of irritability and hyperactivity, with improvements in physical growth, sleep and social interactions. Consistent with potential effects on lipid metabolism, we found that females with AS had altered levels of three members of the apolipoprotein family (APOA1, APOC2, APOE), which are involved in cholesterol transport. There is extensive evidence that the levels of some apolipoproteins are disturbed in ASC and other conditions [28]. The present study suggests these effects may be more prominent in females with ASC. This is also in line with other studies which have shown that APOA1 levels are reduced in women with polycystic ovary syndrome (PCOS), which has an increased prevalence in ASC relative to the general population [30].

We also found female-specific changes in ADIPO, GLCE, FETUB and SHBG, which all have functions related to lipid biosynthesis or metabolism [31-34]. In addition to its role in lipid-related pathways, SHBG also serves as the main transport protein for sex steroids such as oestrogen and testosterone [35]. According to the foetal androgen theory, high levels of testosterone and other steroid androgens during prenatal brain development can contribute to the development of ASC [16]. Previous studies have demonstrated that there is a correlation between high foetal testosterone concentrations with evidence of more masculinisedbehaviour in later life [16] and an increased number of autistic traits in the normal population [36]. Our finding of elevated levels of SHBG in females may indicate higher levels of free testosterone. This is of potential interest as several studies have found that administration of drugs such as statins can reduce the effects of hyperandrogenemia in conditions such as PCOS that are associated with high testosterone levels [37-39]. One report has hypothesized that statins may be a potential novel therapy in autism and epilepsy [40]. In addition, insulin-sensitizing agents appear to be efficacious in reducing testosterone levels and alleviating other symptoms of PCOS [41]. If such treatments are effective for ASC, the present results suggest that it may be more appropriate to apply these specifically in the case of females with AS. However, caution is needed in extrapolating from the current results as these are derived from adults and we do not know if these would hold for younger individuals. Nor do we make claims for treatment implications as side-effects have yet not been fully evaluated.

Most of the remaining proteins changes specifically in females identified by the combined proteomic profiling platforms are involved in regulation of cell growth, 
differentiation, survival or apoptosis [42-48]. This included changes in CHGA, EPO, PAP, PTPA, TLE1, RN149 and TRIPB. The others have roles in immune system function (IgA, IL-3, TENA, CLC4K [49,50]), regulation of brainspecific mRNAs (ZC3HE [51]) transfer RNA processing (MRRP1 [52]) or cell adhesion and mobility (ARMC3 [53]). Previous studies have shown a reduction of IgA levels in subgroups of people with ASC although the study design did not test for sex differences [49].

The majority of proteins that showed differences in males with AS specifically were associated with inflammation pathways. These included TNF-alpha, which has previously been identified in children with ASC, although not in a sex specific manner [54]. Effects of inflammation are identified frequently in studies of ASC and this could be due to the high proportion of males that are normally analysed in these studies. For example, previous studies found that ICAM-1 was decreased in autism [55], although we showed that it was increased significantly in adult males with AS. Other inflammation-related proteins that were found to be altered specifically in males with AS were TF, CTGF, IL-16 and IL-12p70 [56-58]. The finding of an inflammatory signature in males may have potential applications for a stratified medical approach. For example, males with AS exhibiting immune dysfunction might be candidates for treatment with anti-inflammatory drugs, subject to normal safety checks. Recently, an open label pilot study showed that treatment with a combination of the flavonoids luteolin and quercetin seemed to be effective in reducing autistic symptoms in children, with no major adverse effects [59]. In addition, a randomised double-blind placebo-controlled trial showed that a combination of risperidone and celecoxib was superior to risperidone alone in treating irritability, social withdrawal, and stereotypy of children with autism [60].

We also found that two proteins associated with other pathways were altered specifically in males with AS. These were BMP6 and RGPD4. BMP6 has been associated previously with proliferation and differentiation of cells [61] and RGPD4 belongs to family of proteins involved in intracellular trafficking and sorting [62]. Finally, six proteins (CHGA, TENA, SHBG, PAP, EPO and IL-3) were changed in both males and females with AS, although these changes occurred in opposite directions. Taken together, these findings provide further evidence for marked differences in the underlying affected pathways between males and females with AS.

There are several limitations to this study. First, there is a potential bias in the molecular classes of the investigated molecules. This is based on the proteins targeted by the multiplexed immunoassay and mass spectrometry platforms that do not cover all functional classes of proteins. Therefore, it is possible that analysis of a different selection of molecules would lead to different conclusions from those drawn in this study. Another limiting factor was the small number of clinical serum samples tested. This was due to the rarity of such samples that could be obtained using strict standard operating procedures from both individuals with AS and matched controls. Also, the fact that this study included only AS individuals who were not on medication could result in a selection bias. For example, this could mean that samples from the less severe cases were tested, such as those without associated anxiety or depression. In addition, as mentioned earlier, the current study has only investigated adults so cannot account for age-related differences that are likely to be important in ASC.

\section{Conclusion}

In conclusion, we have identified sex-specific proteomic changes in sera from adults with AS. Females showed changes in proteins mainly associated with lipid transport and metabolism, including FAI, and males showed changes predominantly in inflammation pathways. Further exploration is warranted into the mechanisms by which these sexually dimorphic molecular phenotypes in AS arise. This may lead to deeper insights into the wellestablished sex differences in the clinical manifestation [63] and brain structure [64] and course of ASC. This may have implications for the development of novel targeted treatment approaches for improved outcomes, and for understanding sex-linked aetiological factors in autism [65].

\section{Additional file}

Additional file 1: Analytes measured using multiplex immunoassay platform.

\section{Abbreviations}

ADIPO: Adiponectin; ANOVA: Analysis of variance; APO: Apolipoprotein; AQ: Autism Spectrum Quotient; ARMC3: Armadillo repeat containing 3; AS: Asperger syndrome; ASC: Autism spectrum condition; BMI: Body mass index; BMP6: Bone morphogenic protein6; CHGA: Chromogranin A; CTGF: Connective tissue growth factor; DSM-IV-TR: The Diagnostic and Statistical Manual of Mental Disorders IV-Text Review; EPO: Erythropoietin; FAl: Free androgen index; FDR: False discovery rate; FETUB: Fetuin B; GABA: Gamma aminobutyric acid; GLCE: Glucuronic acid epimerase; ICAM-1: Intracellular adhesion molecule-1; Ig: Immunoglobulin; IL: Interleukin; IPKB: Ingenuity Pathways Knowledge Database; kDa: KiloDaltons; LC-MSE: Liquid chromatography-mass spectrometry; MAP: Multi-Analyte Profiling; MRRP1: RNA G9 methyltransferase domain cont 1; NMDA: N-methyl-D-aspartate; PAP: Prostatic antigen phosphate; PCA: Principle component analysis; PCOS: Polycystic ovary syndrome; PTPA: Protein phosphatase 2A activator; QTOF: Quadrupole time-of-flight; RGPD4: RANBP2-like and GRIP domain containing 5; RN149: Ring finger protein 149; SHBG: Sex hormone binding globulin; SLOS: Smith-Lemli-Opitz Syndrome; SRM: Selective Reaction Monitoring; STARD: Standards for Reporting of Diagnostic Accuracy; TENA: Tenascin C; TLE1: Transducin-like enhancer of split 1; TNF: Tumour necrosis factor; TRIPB: Thyroid hormone receptor interactor 11; UPLC: Ultra-performance liquid chromatography; ZC3HE: Zinc finger CCCH-type containing 14. 


\section{Competing interests}

SB and JDC are consultants for Myriad-RBM. This does not affect policies regarding sharing of data and materials specified by this journal.

\section{Authors' contributions}

$H S, J M R, P S$ and JDC carried out the molecular profiling data analyses. PCG, SB and SB-C interpreted the results, prepared the figures and tables, and wrote the manuscript. LR, SB, HR and SB-C designed the clinical studies and edited the manuscript. El, LR, and BA coordinated clinical data collection. SB and SB-C conceived the study, interpreted the results and edited the manuscript. All authors read and approved the final manuscript.

\section{Authors' information}

Simon Baron-Cohen and Sabine Bahn are senior authors.

\section{Acknowledgements}

This work was funded by Autism Speaks Grant \#6009, the Dutch Fund for Economic Structure Reinforcement (FES), under grant agreement number 0908 (NeuroBasicPharmaPhenomics project). SBC, BA, El and LR were supported by the MRC UK, the Wellcome Trust, the Autism Research Trust, and the Nancy Lurie Marks Family Foundation during the period of this work, and were part of the NIHRCLAHRC for Cambridgeshire and Peterborough NHS Foundation Trust.

\section{Author details}

${ }^{1}$ Department of Chemical Engineering \& Biotechnology, University of Cambridge, Tennis Court Road, Cambridge, UK. ${ }^{2}$ Autism Research Centre, Department of Psychiatry, University of Cambridge, 18B Trumpington Road, CB2 8AH, Cambridge, UK. ' Division of Child Neurology and Psychiatry, Department of Developmental Neuroscience, Stella Maris Scientific Institute, Pisa, Italy. ${ }^{4}$ Department of Neuroscience, Erasmus Medical Centre, Rotterdam, Netherlands.

Received: 18 October 2013 Accepted: 31 December 2013

Published: 27 January 2014

\section{References}

1. American Psychiatric Association: Diagnostic and Statistical Manual of Menta Disorders IV. Washington DC: American Psychiatric Press Inc; 1994. ISBN-10: 0890420262.

2. Baron-Cohen S, Scott FJ, Allison C, Williams J, Bolton P, Matthews FE, Brayne C: Prevalence of autism-spectrum conditions: UK school-based population study. Br J Psychiatry 2009, 194:500-509.

3. Baird G, Simonoff E, Pickles A, Chandler S, Loucas T, Meldrum D, Charman T: Prevalence of disorders of the autism spectrum in a population cohort of children in South Thames: the Special Needs and Autism Project (SNAP). Lancet 2006, 368:210-215.

4. Woodbury-Smith MR, Volkmar FR: Asperger syndrome. Eur Child AdolesC Psychiatry 2009, 18:2-11.

5. Mattila ML, Kielinen M, Jussila K, Linna SL, Bloigu R, Ebeling H, Moilanen I: An epidemiological and diagnostic study of Asperger syndrome according to four sets of diagnostic criteria. J Am Acad Child Adolesc Psychiatry 2007, 46:636-646.

6. Schwarz E, Guest PC, Rahmoune H, Wang L, Levin Y, Ingudomnukul E, Ruta $L$, Kent L, Spain M, Baron-Cohen S, Bahn S: Sex-specific serum biomarker patterns in adults with Asperger's syndrome. Mol Psychiatry 2011, 16:1213-1220

7. Ramsey JM, Schwarz E, Guest PC, van Beveren NJ, Leweke FM, Rothermundt M, Bogerts B, Steiner J, Ruta L, Baron-Cohen S, Bahn S: Molecular sex differences in human serum. PLoS One 2012, 7:e51504.

8. Venkat A, Jauch E, Russell WS, Crist CR, Farrell R: Care of the patient with an autism spectrum disorder by the general physician. Postgrad Med $J$ 2012, 88:472-481.

9. Levin Y, Hradetzky E, Bahn S: Quantification of proteins using dataindependent analysis (MSE) in simple andcomplex samples: a systematic evaluation. Proteomics 2011, 11:3273-3287.

10. Baron-Cohen S, Wheelwright S, Skinner R, Martin J, Clubley E: The autism-spectrum quotient (AQ): evidence from Asperger syndrome/ high-functioning autism, males and females, scientists and mathematicians. J Autism Dev Disord 2001, 31:5-17.
11. Schwarz E, Guest PC, Rahmoune H, Harris LW, Wang L, Leweke FM, Rothermundt M, Bogerts B, Koethe D, Kranaster L, Ohrmann P, Suslow T, McAllister G, Spain M, Barnes A, van Beveren NJ, Baron-Cohen S, Steiner J, Torrey FE, Yolken RH, Bahn S: Identification of a biological signature for schizophrenia in serum. Mol Psychiatry 2012, 17:494-502

12. Li GZ, Vissers JP, Silva JC, Golick D, Gorenstein MV, Geromanos SJ: Database searching and accounting of multiplexed precursor and product ion spectra from the data independent analysis of simple and complex peptide mixtures. Proteomics 2009, 9:1696-1719.

13. Neubert H, Bonnert TP, Rumpel K, Hunt BT, Henle ES, James IT: Label-free detection of differential protein expression by LC/MALDI mass spectrometry. J Proteome Res 2008, 7:2270-2279.

14. Martins-de-Souza D, Alsaif M, Ernst A, Harris LW, Aerts N, Lenaerts I, Peeters PJ Amess B, Rahmoune H, Bahn S, Guest PC: The application of selective reaction monitoring confirms dysregulation of glycolysis in a preclinical model of schizophrenia. BMC research notes 2012, 5:146.

15. Chang $C Y$, Picotti $P$, Hüttenhain $R$, Heinzelmann-Schwarz $V$, Jovanovic $M$ Aebersold R, Vitek O: Protein significance analysis in selected reaction monitoring (SRM) measurements. Mol Cell Proteomics 2012, 11(4): M111.014662. 10.1074/mcp.M111.014662. [Epub21 Dec 2011].

16. Ingudomnukul E, Baron-Cohen S, Wheelwright S, Knickmeyer R: Elevated rates of testosterone-related disorders in women with autism spectrum conditions. Horm Behav 2007, 51:597-604.

17. Ye H, Liu J, Wu JY: Cell adhesion molecules and their involvement in autism spectrum disorder. Neurosignals 2010, 18:62-71.

18. Richdale AL, Prior MR: Urinary cortisol circadian rhythm in a group of high-functioning children with autism. J Autism Dev Disord 1992, 22:433-447.

19. Jyonouchi $H$, Sun $S$, Le H: Proinflammatory and regulatory cytokine production associated with innate and adaptive immune responses in children with autism spectrum disorders and developmental regression. J Neuroimmunol 2001, 120:170-179.

20. Miyazaki K, Narita N, Sakuta R, Miyahara T, Naruse H, Okado N, Narita M: Serum neurotrophin concentrations in autism and mental retardation: a pilot study. Brain Dev 2004, 26:292-295.

21. Suzuki K, Matsuzaki H, Iwata K, Kameno Y, Shimmura C, Kawai S, Yoshihara Y, Wakuda T, Takebayashi K, Takagai S, Matsumoto K, Tsuchiya KJ, Iwata Y, Nakamura K, Tsujii M, Sugiyama T, Mori N: Plasma cytokine profiles in subjects with high-functioning autism spectrum disorders. PLoS One 2011, 6:e20470.

22. Ashwood P, Krakowiak P, Hertz-Picciotto I, Hansen R, Pessah IN, Van de Water J: Associations of impaired behaviours with elevated plasma chemokines in autism spectrum disorders. J Neuroimmunol 2011 232:196-199.

23. Heuer L, Ashwood P, Schauer J, Goines P, Krakowiak P, Hertz-Picciotto I, Hansen R, Croen LA, Pessah IN, Van de Water J: Reduced levels of immunoglobulin in children with autism correlates with behavioral symptoms. Autism Res 2008, 1:275-283.

24. Hergüner S, Keleşoğlu FM, Tanıdır C, Cöpür M: Ferritin and iron levels in children with autistic disorder. Eur J Pediatr 2012, 171:143-146.

25. Iwata K, Matsuzaki H, Miyachi T, Shimmura C, Suda S, Tsuchiya K, Matsumoto K, Suzuki K, Iwata Y, Nakamura K, Tsujii M, Sugiyama T, Sato K, Mori $\mathrm{N}$ : Investigation of the serum levels of anterior pituitary hormones in male children with autism. Mol Autism 2011, 2:16. doi:10.1186/2040-2392-2-16.

26. Tierney E, Bukelis I, Thompson RE, Ahmed K, Aneja A, Kratz L, Kelley RI: Abnormalities of cholesterol metabolism in autism spectrum disorders. Am J Med Genet B Neuropsychiatr Genet 2006, 141B:666-668.

27. Moses L, Katz N, Weizman A: Metabolic profiles in adults with autism spectrum disorder and intellectual disabilities. Eur Psychiatry 2013 10.1016/j.eurpsy.2013.05.005. [Epub ahead of print].

28. Woods AG, Sokolowska I, Taurines R, Gerlach M, Dudley E, Thome J, Darie CC: Potential biomarkers in psychiatry: focus on the cholesterol system. J Cell Mol Med 2012, 16:1184-1195.

29. Aneja A, Tierney E: Autism: the role of cholesterol in treatment. Int Rev Psychiatry 2008, 20:165-170.

30. Palomba S, Marotta R, Di Cello A, Russo T, Falbo A, Orio F, Tolino A, Zullo F, Esposito R, La Sala GB: Pervasive developmental disorders in children of hyperandrogenic women with polycystic ovary syndrome: a longitudinal case-control study. Clin Endocrinol (Oxf) 2012, 77:898-904.

31. Havel PJ: Control of energy homeostasis and insulin action by adipocyte hormones: leptin, acylation stimulating protein, and adiponectin. Curr Opin Lipidol 2002, 13:51-59. 
32. Hodoğlugil U, Williamson DW, Yu Y, Farrer LA, Mahley RW: Glucuronic acid epimerase is associated with plasma triglyceride and high-density lipoprotein cholesterol levels in Turks. Ann Hum Genet 2011, 75:398-417.

33. Choi JW, Liu H, Mukherjee R, Yun JW: Downregulation of fetuin-B and zinc-a2-glycoprotein is linked to impaired fatty acid metabolism in liver cells. Cell Physiol Biochem 2012, 30:295-306.

34. Bonnet F, Cephise FL, Gautier A, Dubois S, Massart C, Camara A, Larifla L, Balkau B, Ducluzeau PH: Role of sex steroids, intrahepatic fat and liver enzymes in the association between SHBG and metabolic features. Clin Endocrinol (Oxf) 2012, 79:517-522 [Epub ahead of print].

35. Rosner W, Hryb DJ, Khan MS, Nakhla AM, Romas NA: Sex hormone-binding globulin: anatomy and physiology of a new regulatory system. J Steroid Biochem Mol Biol 1991, 40:813-820.

36. Auyeung B, Baron-Cohen S, Ashwin E, Knickmeyer R, Taylor K, Hackett G: Fetal testosterone and autistic traits. $\mathrm{Br} J$ Psychol 2009, 100:1-22.

37. Schooling CM, Au Yeung SL, Freeman G, Cowling BJ: The effect of statins on testosterone in men and women, a systematic review and metaanalysis of randomized controlled trials. BMC Med 2013, 11:57.

38. Sathyapalan T, Kilpatrick ES, Coady AM, Atkin SL: The effect of atorvastatin in patients with polycystic ovary syndrome: a randomized double-blind placebo-controlled study. J Clin Endocrinol Metab 2009, 94:103-108.

39. Banaszewska B, Pawelczyk L, Spaczynski RZ, Dziura J, Duleba AJ: Effects of simvastatin and oral contraceptive agent on polycystic ovary syndrome: prospective, randomised, crossover trial. J Clin Endocrinol Metab 2007, 92:456-461. Epub 2006 Nov 14.

40. Ghanizadeh A: May lovastatin target both autism and epilepsy? A novel hypothesized treatment. Epilepsy Behav 2011, 20:422.

41. Hwang KR, Choi YM, Kim JJ, Chae SJ, Park KE, Jeon HW, Ku SY, Kim SH, Kim JG, Moon SY: Effects of insulin-sensitizing agents and insulin resistance in women with polycystic ovary syndrome. Clin Exp Reprod Med 2013, 40:100-105.

42. Chromogranin A, Duque M, Modlin IM, Gupta A, Saif MW: Biomarkers in neuroendocrine tumors. JOP 2013, 14:372-376.

43. Chin H, Saito T, Arai A, Yamamoto K, Kamiyama R, Miyasaka N, Miura O: Erythropoietin and IL-3 induce tyrosine phosphorylation of CrkL and its association with Shc, SHP-2, and Cbl in hematopoietic cells. Biochem Biophys Res Commun 1997, 239:412-417.

44. Chu TM: Prostate cancer-associated markers. Immunol Ser 1990, 53:339-356.

45. Janssens V, Goris J: Protein phosphatase $2 A$ : a highly regulated family of serine/threonine phosphatases implicated in cell growth and signalling. Biochem J 2001, 353:417-439.

46. Dastidar SG, Narayanan S, Stifani S, D'Mello SR: Transducin-like enhancer of Split-1 (TLE1) combines with Forkhead box protein G1 (FoxG1) to promote neuronal survival. J Biol Chem 2012, 287:14749-14759.

47. Hong SW, Jin DH, Shin JS, Moon JH, Na YS, Jung KA, Kim SM, Kim JC, Kim KP, Hong YS, Lee JL, Choi EK, Lee JS, Kim TW: Ring finger protein 149 is an E3 ubiquitin ligase active on wild-type v-Raf murine sarcoma viral oncogene homolog B1 (BRAF). J Biol Chem 2012, 287:24017-24025.

48. Smits $P$, Bolton AD, Funari V, Hong M, Boyden ED, Lu L, Manning DK, Dwyer ND, Moran JL, Prysak M, Merriman B, Nelson SF, Bonafé L, Superti-Furga A, Ikegawa S, Krakow D, Cohn DH, Kirchhausen T, Warman ML, Beier DR: Lethal skeletal dysplasia in mice and humans lacking the golgin GMAP-210. N Engl J Med 2010, 362:206-216.

49. Warren RP, Odell JD, Warren WL, Burger RA, Maciulis A, Daniels WW, Torres AR: Brief report: immunoglobulin A deficiency in a subset of autistic subjects. J Autism Dev Disord 1997, 27:187-192.

50. Dalkowski A, Schuppan D, Orfanos CE, Zouboulis CC: Increased expression of tenascin C by keloids in vivo and in vitro. Br J Dermatol 1999, 141:50-56.

51. Kelly S, Pak C, Garshasbi M, Kuss A, Corbett AH, Moberg K: New kid on the ID block: neural functions of the $\mathrm{Nab} 2 / \mathrm{ZC} 3 \mathrm{H} 14$ class of $\mathrm{Cys}_{3} \mathrm{His}$ tandem zinc-finger polyadenosine RNA binding proteins. RNA Bio/ 2012, 9:555-562.

52. Swinehart WE, Henderson JC, Jackman JE: Unexpected expansion of tRNA substrate recognition by the yeast $\mathrm{m} 1 \mathrm{G} 9$ methyltransferase Trm10. RNA 2013, 19:1137-1146.

53. Li X, Liu B, Ji CN, Kang Y, Mao Y: Cloning and expression of ARMC3_v2, a novel splicing variant of the human ARMC3 gene. Genetika 2006, 42:999-1003.

54. Croonenberghs J, Bosmans E, Deboutte D, Kenis G, Maes M: Activation of the inflammatory response system in autism. Neuropsychobiology 2002, 45:1-6.
55. Kameno Y, Iwata K, Matsuzaki H, Miyachi T, Tsuchiya KJ, Matsumoto K, Iwata Y, Suzuki K, Nakamura K, Maekawa M, Tsuji M, Sugiyama T, Mori N: Serum levels of soluble platelet endothelial cell adhesion molecule- 1 and vascular cell adhesion molecule-1 are decreased in subjects with autism spectrum disorder. Mol Autism 2013, 4:19.

56. van den Hengel LG, Versteeg HH: Tissue factor signalling: a multi-faceted function in biological processes. Front Biosci (Schol Ed) 2011, 3:1500-1510.

57. Dendooven A, Gerritsen KG, Nguyen TQ, Kok RJ, Goldschmeding R: Connective tissue growth factor (CTGF/CCN2) ELISA: a novel tool for monitoring fibrosis. Biomarkers 2011, 16:289-301.

58. Singh VK: Plasma increase of interleukin-12 and interferon-gamma. Pathological significance in autism. J Neuroimmunol 1996, 66:143-145.

59. Taliou A, Zintzaras E, Lykouras L, Francis K: An open-label pilot study of a formulation containing the anti-inflammatory flavonoid luteolin and its effects on behavior in children with autism spectrum disorders. Clin Ther 2013, 35:592-602.

60. Asadabadi M, Mohammadi MR, Ghanizadeh A, Modabbernia A, Ashrafi M, Hassanzadeh E, Forghani S, Akhondzadeh S: Celecoxib as adjunctive treatment to risperidone in children with autistic disorder: a randomized, double-blind, placebo-controlled trial. Psychopharmacology (Berl) 2013, 225:51-59.

61. Gosselet FP, Magnaldo T, Culerrier RM, Sarasin A, Ehrhart JC: BMP2 and BMP6 control p57(Kip2) expression and cell growth arrest/terminal differentiation in normal primary human epidermal keratinocytes. Cell Signal 2007, 19:731-739.

62. Man Z, Kondo Y, Koga H, Umino H, Nakayama K, Shin HW: Arfaptins are localized to the trans-Golgi by interaction with Arl1, but not Arfs. J Biol Chem 2011, 286:11569-11578.

63. Lai MC, Lombardo MV, Pasco G, Ruigrok AN, Wheelwright SJ, Sadek SA, Chakrabarti B, MRC AIMS Consortium, Baron-Cohen S: A behavioural comparison of male and female adults with high functioning autism spectrum conditions. PLOS One 2011, 6:e20835.

64. Lai MC, Lombardo MV, Suckling J, Ruigrok AN, Chakrabarti B, Ecker C, Deoni SC, Craig MC, Murphy DG, Bullmore ET, MRC AIMS Consortium, Baron-Cohen S: Biological sex affects the neurobiology of autism. Brain 2013, 136:2799-2815.

65. Baron-Cohen S, Lombardo MV, Auyeung B, Ashwin E, Chakrabarti B, Knickmeyer $\mathrm{R}$ : Why are autism spectrum conditions more prevalent in males? PLOS Biol 2011, 9:e1001081.

doi:10.1186/2040-2392-5-4

Cite this article as: Steeb et al:: Serum proteomic analysis identifies sex-specific differences in lipid metabolism and inflammation profiles in adults diagnosed with Asperger syndrome. Molecular Autism 2014 5:4.

\section{Submit your next manuscript to BioMed Central and take full advantage of:}

- Convenient online submission

- Thorough peer review

- No space constraints or color figure charges

- Immediate publication on acceptance

- Inclusion in PubMed, CAS, Scopus and Google Scholar

- Research which is freely available for redistribution 